\title{
A Framework for Real-time Pro-active Safety Assistance for Mobile Crane Lifting
}

4

$7{ }^{1} \mathrm{PhD}$ student, School of Civil and Environmental Engineering, Georgia Institute of Technology,

8790 Atlanta Dr. N.W., Atlanta, GA 30332-0355, USA; email: yihaifang@ gatech.edu

$9{ }^{2}$ Associate Professor, School of Civil and Environmental Engineering, Georgia Institute of

10 Technology, 790 Atlanta Dr. N.W., Atlanta, GA 30332-0355, USA; email:

11 yong.cho@ce.gatech.edu (*Corresponding Author)

$12{ }^{3} \mathrm{PhD}$ student, School of Electrical and Computer Engineering, Robotics Institute, Georgia

13 Institute of Technology, 777 Atlanta Dr. N.W., Atlanta, GA 30332-0355, USA; email:

14 jchen490@gatech.edu

15

16

17

18

19

20

21

22

23

(C) 2016. This manuscript version is made available under the Elsevier user license http://www.elsevier.com/open-access/userlicense/1.0/ 


\section{ABSTRACT}

25 Despite many safety considerations addressed in lift pre-planning, the ability to provide real-time

26 safety assistance to crane operators and to mitigate human errors during the lifting operation is

27 missing. This research developed a framework for an operation-level safety assistance system

28 enabled by continuous crane motion capturing, pro-active hazard analysis, and real-time 3D

29 visualization. A sensor system captures the critical motions of crane parts, based on which the

30 entire crane motions are reconstructed in real-time with as-is lift site conditions modeled based

31 on point cloud data. The risk of colliding the crane parts and lifted load to surrounding

32 obstructions is analyzed and warnings are provided to the operator through a user interface. A

33 prototype system was developed based on the framework and deployed on a mobile crane. Field

34 test results indicate that the system can accurately reconstruct crane motion in real-time and 35 provide pro-active warnings that allow the operator to make timely decisions to mitigate the risk.

\section{KEY WORDS}

38 Crane safety; human error; real-time; crane motion capturing; collision hazard analysis; 3D

39 reconstruction; point cloud; sensor. 
48 A crane is one of the most essential and commonly used type of machinery in the construction 49 industry. In the United States alone, approximately 125,000 cranes of different types are in

50 operation everyday among all sectors of the construction industry, from single houses to

51 international mega projects [1]. Throughout the entire construction phase of a project, cranes are

52 responsible for a great portion of vertical and horizontal transportations of construction resources

53 including material, equipment, and personnel. Lifting operations are inherently a sophisticated

54 job given that the crane is a highly complex piece of machinery that requires extensive

55 experience and skill to operate. For example, crane parts (e.g., boom, jib, trolley, hook) operate

56 independently based on different modes of motion (e.g., swing, translate, lift). Although

57 manipulated individually, every component is interrelated with one another implicitly. Every

58 single motion of one component may affect the movement of other crane parts and the stability 59 of the load.

A crane operator is one of the most well paid jobs in the construction industry because of

61 not only extensive skills and experience required but also the risks and responsibility associated

62 with crane operations. Unsurprisingly, cranes are considered the most dangerous piece of

63 equipment on construction sites by general site employees [2]. Given the magnitude of crane

64 weight and the huge workspace cranes occupy, the potential loss of property and life due to

65 incidents related to crane is tremendous. From 1992 to 2006, crane-related accidents in the

66 United States led to 632 worker deaths in 610 cases, an average of 43 deaths per year [3].

67 Statistics from Bureau of Labor Statistics (BLS) reveals that from 1997 to 2013, 1171 fatalities

68 were observed in crane-related accidents in all industry sectors. Unfortunately, the construction

69 industry alone was responsible for 544 fatalities in the period, which accounts for almost half of 
the deaths in all industry sectors combined. It should be noted that mobile cranes, as the most

71 commonly used type of crane in construction industry, are responsible for $70 \%$ of these accidents

72 [3]. Unlike other accidents on construction sites, the victims in crane-related accidents are not

73 necessarily limited to construction labors and crane operators but also pedestrians as observed in 74 many crane-related accidents [4]. fully investigate and uncover them. The Center for Construction Research and Training (CPWR) revealed that $61 \%$ of 632 fatalities involved crane parts or loads colliding with obstructions (e.g.,

78 power lines, personnel, structure) [3]. Based on the crane accident data from the Occupational

79 Safety and Health Administration (OSHA) from 1997 to 2003, Beavers et al. concluded that 80 struck by load (32\%) and failure of boom/cable (12\%) together accounted for $44 \%$ of the total 81 number of fatalities [2]. It should be noted that $24 \%$ of these fatalities occurred during 82 lifting/moving equipment and materials, being the second to mobilization (31\%). In most cases, 83 the "struck by" accidents occur mainly due to spatial conflicts existing between crane parts and 84 the surrounding obstructions. Within the large workspace of cranes, the presence of built 85 building structure, storage of materials, and power lines all induce potential spatial conflicts 86 during crane lifting operations. Suruda et al. [5] reported that $40 \%$ of the deaths in crane-related 87 accidents were related to spatial conflicts. Furthermore, when analyzing crane-related spatial conflicts, one must consider the dynamic crane motions and changes in the surrounding environment as the project proceeds [6].

91 phase, crane safety in the construction phase mainly relies on crane operator skills and 92 experience. Operator proficiency was considered the highest degree of influence that will affect 
crane safety by construction equipment and safety experts [7]. King found that 32 out of 75 crane accidents from 2004 to 2010 (43\%) were due to the operator failure in their responsibilities [8]. Neitzel et al. pointed out that the crane operator has the most direct influence on the safety of crane lifting operations, and thus they must have the technical and performance proficiency in operating cranes [4]. Operator errors occur because of human frailties such as distraction or fatigue, or because of misjudgement due to lack of experience or inadequate time to react to an unexpected event [7]. Since many construction activities are highly dependent on crane rigging/lifting for material delivery and structure erection, crane operators usually start their work much earlier than other crews. Crane operators, therefore, would be generally more fatigued and thus more prone to making errors when it approaches the end of the day. In addition, crane operators operate huge machines with loads based on limited information obtained from other labor, typically the riggers or signalmen [9]. Construction sites are often very congested with materials, equipment and structures of different heights and shapes. These obstructions introduce massive blind spots into crane lifting operations. Sitting high in a tower crane cabin or low in a mobile crane cabin, operators often find it difficult to determine the absolute height or relative distances between the lifted load and surrounding objects. The limited visibility and poor depth perception lead to the poor situational awareness of the hazards associated with the lift operation, which greatly limits their abilities to identify potential hazards, choose risk-free paths, and timely react to emergencies [10]. It has been well recognized that real-time information and assistance for decision making are necessary and sometimes critical for many construction tasks involving human-machine interaction [11].

In order to improve the operators' situational awareness to the potential hazards associated with crane lifting, this research developed a framework of a safety assistance system 
116 for mobile crane lift operations enabled by real-time crane motion monitoring, pro-active hazard

117 analysis and 3D visualization. This paper starts with a thorough review of current lifting safety

118 practices and technologies. Then, a framework of the pro-active safety assistance system is

119 introduced with details in sensory systems, lift site modeling and updating, and hazard analysis

120 and visualization. The proposed framework is then demonstrated with the prototype pro-active

121 safety assistance system deployed on a telescopic boom mobile crane. Based on the collected

122 field test data, the performance of the prototype system is evaluated with respect to

123 reconstruction accuracy, latency, and ease of use. Finally, the potentials and limitations of the

124 proposed framework approach are discussed.

\section{RELATED WORK}

\section{2.1. Crane Lift Planning}

128 Crane lifting planning is an essential planning mission that is critical for the productivity and 129 safety of the entire project [12]. Tasks in crane lifting planning usually include two basic 130 components: crane location planning and lifting path planning. The crane location or layout 131 planning aims to maximize crane utilization in transporting construction resources. This is a 132 complicated optimization problem involving various spatial-temporal constraints. In current

133 practices, a crane location is typically determined by experienced lift coordinators through trial 134 and error, given the site shape and topography, and the distribution of estimated lifting tasks [13].

135 This manual process can be assisted or validated by simulation technologies [14] [15] and 136 optimization algorithms [16] [17][18][19].

137 The goal of path planning is to plan a collision-free and efficient lift path based on load 138 data (e.g., size, weight) and site geometric constraints. Traditionally, the lift path is planned 
139 mainly depending on the planners' intuition and experience and this process is often labor

140 intensive and error prone [20]. Recently, researchers have adopted the knowledge and techniques

141 in robotic motion planning to automate the lift planning process. In this approach, a crane is

142 treated as a multi degree-of-freedom robotic manipulator and various algorithms (e.g.,

143 Probabilistic Road Map [21], Rapidly Exploring Random Tree (RRT) [22][23][24][25]) were

144 tested to optimize a lift path to ensure a collision-free travel while guaranteeing a respectively

145 short lift path. It should be noted that the computer-aided path planning only works when

146 provided with accurate and comprehensive site geometric information. Site geometry, however,

147 is not always available or accurate in the early planning phase. In addition to static obstructions,

148 when analyzing crane-related spatial conflicts, one must consider the dynamic crane motions and

149 changes in the surrounding environment as the project proceeds [6]. Design changes occur

150 throughout the planning phase and even during the construction phase, and thus site drawings or

151 models used for lift path planning no longer represent all the geometric constraints existing in the

152 actual lift site. These variances between planned and actual site conditions inevitably

153 compromise the validity of the planned lift paths.

\subsection{Real-time Lift Safety Assistance}

156 With the development of information technology, researchers realized that technology can

157 provide another layer of protection in construction safety [26]. Crane operations can benefit from

158 technology similar to the advanced driver assistance systems implemented on vehicles that

159 provide real-time support to the driver based on surrounding situations. The following sections

160 introduce three technologies that have been investigated or implemented in crane lifting

161 operations. 
163 A load moment indicator (LMI) is a system that aids the crane operator by sensing (directly or

164 indirectly) the overturning moment on the crane, i.e., load multiplied by radius [27]. It compares

165 the current lifting condition to the crane's rated capacity or load chart, and indicates to the

166 operator the percentage of capacity at which the equipment is working. This system has become

167 standard in heavy-duty cranes from many crane manufacturers and its performance in the field

168 has proved its effectiveness in preventing accidents due to overloading. However, the LMI

169 system cannot address other hazards associated to crane operation such as collisions and

170 excessive load sway.

\section{2.2.2. Camera system}

173 Using a camera is a simple solution for enhancing the visibility and situational awareness of

174 crane operators. Typically, a camera system involves a wireless camera mounted on crane trolley

175 or hook block directed downwards, and a monitor in the crane cabin (sometimes also in the

176 management office) showing the lifted load and the workspace underneath. Lee et al. [28]

177 proposed a tower crane motion monitoring system equipped with wireless video camera and

178 LCD cabin monitor enabled by a wireless local area network (WLAN). The system provides the

179 crane operator with an enhanced view of the workspace and the lifted load. Shapira et al. [29]

180 developed a vision system for tower cranes to increase the operators' visibility to the load during

181 loading and unloading. Based on the feedback from domain experts, it was concluded that the

182 vision system offers potential benefits in safety enhancement and productivity improvement

183 especially during blind lifts and lifts in the proximity of obstructions. In addition to monitoring 
184 the lifted load, Putnik et al. [30] proposed a more comprehensive system with multiple cameras 185 streaming from different angles to cabin monitors.

186 Camera-based methods are effective in terms of providing alternative views to enhance 187 operator visibility and situational awareness. However, the monitor in the cabin provides only 2188 dimensional images without depth perception. This means the operator cannot determine the 189 height of load at the lower level or the proximity from the load to surrounding objects. Despite 190 the function of zooming in/out, cameras are fixed to the crane parts that makes it less flexible in 191 complicated lifting scenarios such as blind lifts. In addition, the attachments including sheath 192 blocks, hooks, slings, and loads can block the camera views. Furthermore, camera systems 193 provide no automatic warning function, and thus the operator has to constantly check the monitor 194 while operating the crane. This introduces additional mental workload and distractions to the 195 operator, which could ironically increase the risk of accidents.

\subsubsection{Real-time location system}

198 A real-time location system (RTLS) is a technology that automatically identifies and tracks the

199 location of objects in real-time. In construction, it has been used for various applications such as

200 site security, resource location tracking, and equipment safety. Zhang et al. employed a high-

201 precision RTLS technology named Ultra-wide band (UWB) for estimating mobile crane poses in 202 near real-time [31]. In this system, UWB readers were deployed around the lifting scene and 203 UWB tags were mounted on different spots of crane boom and lifted objects. The system is able 204 to estimate crane poses in near real-time through noise filtering and missing data filling. Using 205 the same technology, Hwang [32] explored methods for tower crane collision prevention. He 206 studied the characteristics of different types of collisions and developed a computer program for 
monitoring crane motions and sending warnings if a potential collision hazard was detected. Li et

208 al. took advantage of Global Positioning System (GPS) and Radio Frequency Identification

209 (RFID) for developing a real-time crane motion monitoring system [33]. Tracking crane parts

210 and construction workers, this system aims to assist the safety operation in blind lifts by

211 detecting the presence of unauthorized workers within a risk zone.

212 These efforts demonstrated the feasibility of using RTLS for crane pose/motion

213 monitoring. However, the RTLS technology suffers from several limitations that compromise its

214 performance in the application of crane motion monitoring. Firstly, most RTLS system requires

215 setting up hardware including readers, cables for data transmission and synchronization, power

216 supply, and computer for data collection and processing. Depending on the range of the RTLS

217 readers, the number of readers needed to cover the site needs to increase accordingly as the size

218 of the crane to be monitored increases. In addition, crane lifting is a 3-dimentional operation that

219 requires the RTLS system to be deployed at various heights in order to tracking the vertical

220 movement of crane parts and load. This not only requires setting up a big amount of RTLS

221 antennas around the lift site ahead of time, but also means a big investment in hardware given

222 that the cost of UWB is approximately $\$ 140 / \mathrm{m}^{2}$ [34]. Secondly, the site security and vandalism

223 for the installed sensing system would be another concern unless the components are installed

224 and retrieved every day. Thirdly, the RTLS technology inherently suffers from signal

225 interference due to electromagnetic interferences, multi-path propagation, fading and scattering

226 of signals [35]. This problem becomes more significant in metallic and densely packed

227 construction environment. As recognized by Zhang et al. [31], their UWB tracking system failed

228 to reliably track the load position because the tags on the crane load cannot be detected and

229 tracked continuously due to serious signal loss. 


\subsection{Direct Motion Measurement}

231 Another method that has been extensively investigated for crane motion monitoring is integrating

232 sensors of different types to capture critical crane motions. Critical crane motions stand for the

233 key freedoms of major crane parts that, when combined are able to represent most possibilities of

234 crane maneuvers. Lee et al. developed a laser-based lifting path tracking method for a robotic

235 crane system to improve the crane lifting productivity [36]. This system employs multiple

236 sensors including a laser device for measuring the load elevation, and an encoder and an

237 accelerometer for measuring horizontal and vertical angles of crane boom. A major limitation of

238 this system is that it does not consider the load sway and, as pointed out by the authors, the

239 configuration of laser sensor and reflection board cannot reliably measure the load elevation

240 during excessive load sway. Advanced from the previous attempt, Lee et al. augmented the crane

241 motion monitoring system by introducing multiple sensors and a BIM-based method for

242 incorporating the surrounding environment [37]. They further analyzed the ease of use and

243 usefulness of the developed system. After 71 days of implementation on a tower crane, the

244 operators heavily relied on the developed system (93.3\%) compared to traditional load moment

245 indicator (LMI) system. However, the operator had to use a 19-key mini keypad to change the

246 crane view angle on the user interface, which very likely compromises the lifting safety and

247 efficiency, as the operator is distracted from the main task. Ren and Wu developed a real-time

248 anti-collision system for mobile cranes that creates a safe lift zone during lifting based on the

249 location and shape of static obstacles collected prior to the lift [38]. However, this process

250 involves extensive manual input and additional data collection time prior to the lift.

Compared to the RTLS-based and camera-based crane monitoring methods, the direct

252 sensor-based solutions hold several apparent advantages. Firstly, hardware employed in sensor- 
253 based systems is usually more cost-effective and durable in long-term use. Once securely

254 installed on the crane, the sensors require little maintenance efforts and with proper enclosure

255 they can work properly in harsh environmental conditions. Secondly, sensor-based methods do

256 not require external hardware deployment on the site, and thus it introduces minimal interruption

257 to other construction activities. Thirdly, sensors-based system can captures crane motions more

258 effectively and comprehensively.

\section{3. METHODOLOGY}

261 Despite several research efforts that have been devoted to improving crane lift safety in pre-

262 planning and execution phases, very few of them can provide crane operators with effective

263 information to enable real-time operational assistance and pro-active hazard warnings. To fill

264 this gap in knowledge, this research designed and created a novel framework that enables pro-

265 active safety assistance for mobile crane lift operations through the integration of real-time

266 motion capturing, rapid lift scene updating, pro-active safety analysis, and visual and audio

267 warnings. As shown in Figure 1, the proposed framework consists of three major components:

268 critical motion capturing, site condition modeling, and hazard analysis and visualization. Real-

269 time actions are indicated by boxes in solid line, which include crane motion capturing and

270 hazard analysis and visualization. Pre-lift actions include crane model preparation and as-is site

271 point cloud acquisition. The following sections introduce each component in the framework in 272 detail. 
Critical motion capturing

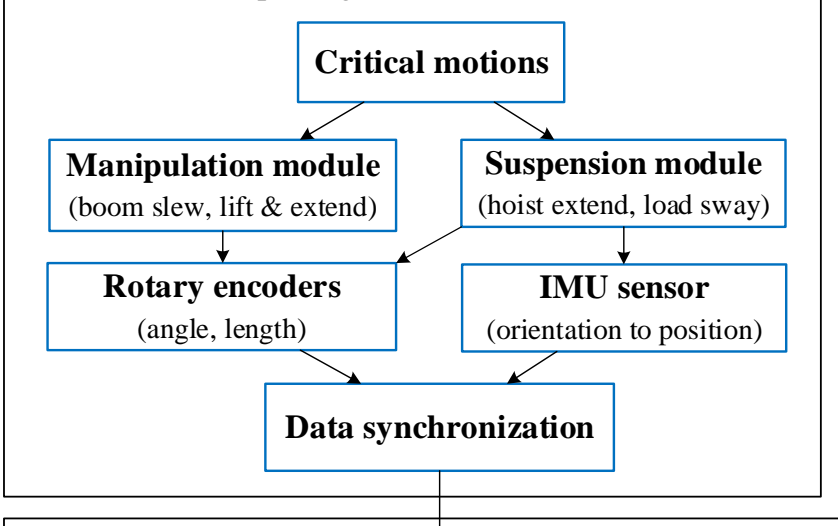

Site condition modeling
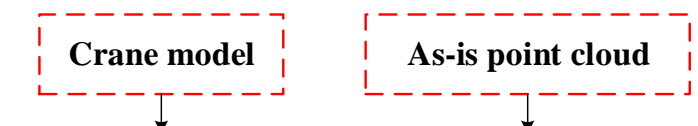

।

Crane model animation | Obstruction recognition |

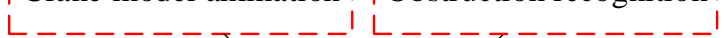

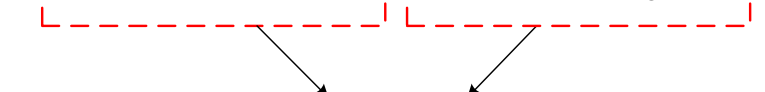

Hazard Analysis \& Visualization

Interactive $3 \mathrm{D}$ visualization

(voice-control)

Real-time safety assistance

(visual \& auditory warning)

Figure 1: A framework for real-time pro-active safety assistance for crane lifting operation

\subsection{Critical Motion Analysis of Crane Manipulation and Suspension Modules}

A crane is comprised of multiple rigid bodies connected by different types of joint, depending on

the crane type and capacity. In this study, the type of telescopic-boom mobile cranes was

selected as they usually have more degrees of freedom than others (e.g., tower cranes or lattice

boom mobile cranes), and thus the design of mobile crane motion capturing proposed in this

research can be easily altered to apply to other types of cranes. A telescopic mobile crane can be

decomposed to two independent modules: the manipulation module and the suspension module

(see Figure 2). The manipulation module is comprised of three rigid bodies including a truck

base, a crane body, and a telescopic boom. Then, the telescopic boom is further broken down 
289 and the crane body as well as the connection between the crane body and the main boom are

290 modeled by a revolute joint that only allows one rotational degree of freedom between the two

291 rigid bodies. The suspension module consists of a normal rigid body, the lifted load, and an

292 extensible rigid body for the hoist line. Although hoist line itself is elastic and thus can be hardly

293 considered a rigid body, when used in a pulley system where double (four cables) or triple tackle

294 (six cables) serve together for lifting, the cable bundle is actually resistant to twisting and 295 bending, especially when the lifted load performs a 3-dimentional pendulum motion in a very

296 small magnitude (less than 10 degree). The connection between the suspension module and the

297 sub-booms is modeled by a spherical joint that allows rotation in all direction and only

298 constraints translational movement. Based on the critical motion analysis, a telescopic boom

299 mobile crane is simplified as six rigid bodies connected by five joints. This means that any

300 motion that can be possibly performed by a crane is able to be kinematically represented by

301 measuring the boom slew angle, boom lift angle and boom extension length in the manipulation

302 module and the length of the hoist line and load sway in the suspension module. 

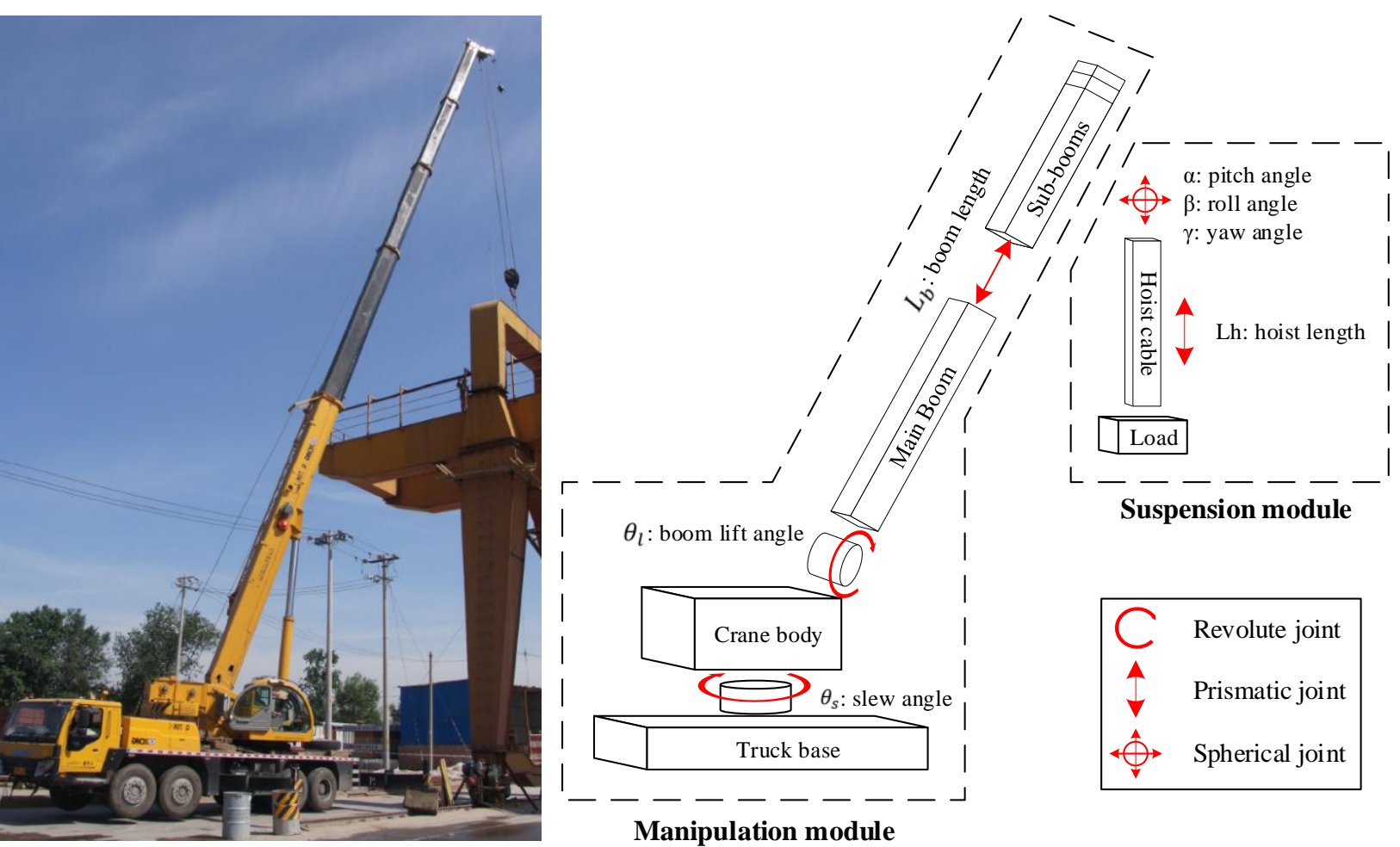

Figure 2: Kinematics configuration of a typical telescopic boom mobile crane

\subsubsection{Manipulation module motion capturing using rotary encoders}

308 Rotary encoders are electromechanical devices that are used in various electronic and mechanical

309 devices for measuring angular position or motion of a shaft or axle in the device. Rotary

310 encoders can be classified into incremental and absolute encoders, or mechanical, optical, and

311 magnetic encoders based on how the digital code is produced [39]. For typical magnetic absolute

312 encoders, strips of magnetized material are placed on the rotating disc and are sensed by a Hall-

313 effect sensor or magneto resistive sensor. The prototype system adopted rotary magnetic absolute

314 encoders to measure three critical motions of the crane manipulation module: boom slew and lift

315 angles, hoist line extension and boom extension. The boom lift angles can also be measured by

316 an inclination sensor alternatively. Although the proposed motion capturing method adopts

317 commercially available encoders, it is more flexible than other commercial solutions [40] in that 
318 the selection and deployment of the sensors are also suitable for retrofitting existing cranes in

319 service.

\subsubsection{Suspension module motion capturing using an inertia measurement unit}

322 An inertial measurement unit (IMU) is an electronic device that measures velocity, orientation,

323 and gravitational forces, using a combination of accelerometers and gyroscopes, sometimes

324 magnetometers. IMU sensors were originally developed for the maneuver of aircraft and

325 spacecraft, such as an unmanned aviation vehicle (UAV) and satellites, to report inertial

326 measurements to the pilot system. Recently, IMU sensors have been widely used as orientation

327 sensors for measuring human body motions in sport athlete training and movie production. A

328 typical IMU sensor contains angular and linear accelerometers for tracking the changes in

329 position and gyroscopes for maintaining an absolute angular reference. Generally, an IMU sensor

330 has least one accelerometer and one gyroscope for each of the three axes: pitch (nose up and

331 down), yaw (nose left and right) and roll (clockwise or counter-clockwise). When rigidly

332 mounted to an object, the IMU sensor measures the linear and angular acceleration and

333 automatically calculates the orientation of the attached object. In the particular case of load sway,

334 it is assumed that the cable length is known and the cable is rigid. Therefore, the load sway

335 motions can be simplified to a typical 3-dimentional (3D) pendulum motion (Figure 3a). Given

336 the measured angular orientation on each axis (Figure 3b) and the cable length, the estimated

337 position of the load relative to the fixed point can be calculated by converting the Euler angle

338 measurements to Cartesian coordinates in the local coordinate system (Figure 3c). 


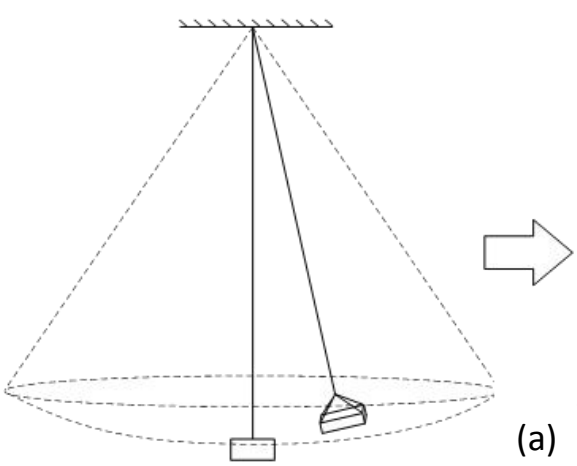

(a)

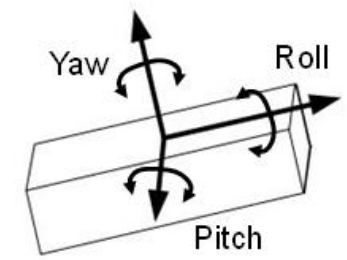

(b)

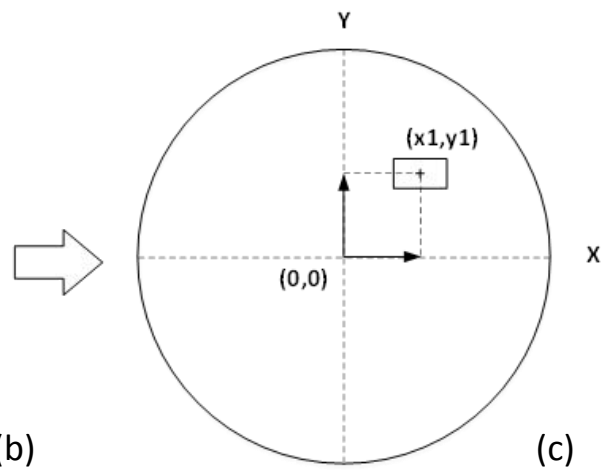

340

341

Figure 3: Transforming the augular measurements to absolute positions (adapted from [41])

This process of converting the Euler angle measurements to Cartesian coordinates is demonstrated in the following steps. Firstly, one single load orientation measurement can be represented by three independent rotations: yaw, pitch, and roll. The yaw, pitch, and roll rotations can comprehensively represent a 3D rigid body in any orientation. These three independent rotations are defined as follows: a yaw is a counterclockwise rotation of $\alpha$ about the $\mathrm{z}$-axis; a pitch is a counterclockwise rotation of $\beta$ about the $\mathrm{y}$-axis; a roll is a counterclockwise rotation of $\gamma$ about the $\mathrm{x}$-axis. Therefore, a single rotation matrix $R(\alpha, \beta, \gamma)$ can be formed by multiplying the yaw, pitch, and roll rotation matrices.

Since the load sway motion is a simple 3-dimentional pendulum motion, the load trajectory actually lies on the internal surface of a virtual sphere with the radius of the cable length. Hence, the unit vector on a local z-axis always points to the center of the sphere. Therefore, converting the Euler angle measurements to Cartesian coordinates is simplified by converting this unit vector on the local z-axis to a vector on global coordinate system according to the rotation matrix containing the three independent rotations. Thus, the load position can be estimated by multiplying the rotation matrix with the unit vector $(0,0,1)$ and the length of the hoist line L using Eq. (1). 
$358 P(x, y, z)=R(\alpha, \beta, \gamma) \cdot\left[\begin{array}{l}0 \\ 0 \\ 1\end{array}\right] \cdot L=\left[\begin{array}{c}\cos \alpha \cdot \sin \beta \cdot \cos \gamma+\sin \alpha \cdot \sin \gamma \\ \sin \alpha \cdot \sin \beta \cdot \cos \gamma-\cos \alpha \cdot \sin \gamma \\ \cos \beta \cdot \cos \gamma\end{array}\right] \cdot L$

It should be noted that the initial orientation measurement $(\alpha, \beta, \gamma)$ is not necessarily the

361 origin since the surface where the sensor is placed might not be completely leveled. Therefore, a

362 deviation from the origin can be expected in the estimation of the load trajectory based on this

363 method. To minimize this error, the initial load orientation is taken into consideration. The

364 orientation data measured when the load is static is averaged and introduced to the calculation as 365 an offset for correcting the deviation.

367 3.2. Site Condition Modeling

\subsubsection{Crane modeling}

369 Cranes are different in types, sizes, and capacities, and thus it is important for the lift assistance system to model the critical crane configurations according to the actual crane geometry and

371 movements. This can be accomplished by modifying an existing crane model according to

372 specifications of the actual crane that will be used for the job. Key dimensions to be considered

373 include the sizes of crane base and cabin, limits of boom length, pivot point of crane's

374 superstructure, and the size and shape of the lifted load.

\subsubsection{Site condition modeling and updating from Point cloud}

377 Cranes' lift capability and maneuverability are greatly affected by the geometric constraints in

378 the surrounding environment, especially for mobile cranes as their location and workspace

379 change quite frequently as a project proceeds. Therefore, it is necessary to integrate lift site

380 environment data into the lift assistance framework. A major limitation in traditional lift 
381 planning approaches is the lack of information to represent the as-is lift site condition. Recent

382 advances in rapid geometry data acquisition make it possible to obtain massive 3-dimentional

383 geometric data in a short amount of time with minimal efforts. This is realized in the proposed

384 framework by rapid modeling and updating the lift site in the form of point cloud. A point cloud

385 is a set of points containing coordinate data (XYZ) and color data (RGB) representing the

386 geometry of objects in the captured scene. Obtained from a terrestrial laser scanner or aerial

387 photogrammetry technology, point clouds can be used to represent object geometries or to

388 reconstruct a scene with good accuracy $(+/-2 \mathrm{~mm})$ and efficiency (up to 976,000 point per

389 second) [42].

To reconstruct an as-is lift site, the site point cloud acquired by a laser scanner is

391 converted into bounding box objects prior to the lifting operation to represent site obstructions

392 for the collision hazard analysis in the next step. The as-is lift site model is updated by manually

393 adding dynamic objects such as vehicles and construction materials on the operator user interface.

394 Research in automating the laser scanning-based site modeling and updating [43] [44] is out of

395 the scope of this research, and advancement in this area will be introduced in future publications.

396 The pipeline of obtaining oriented bounding boxes for various objects in point clouds involves

397 the steps of segmentation, clustering, and orientation estimation. The input point cloud is first

398 down-sampled from its original number of points to around 10,000 points by performing voxel

399 grid filtering. Individual points in the original point cloud are projected onto a grid of $1 \mathrm{~cm}$-sized

400 voxels and only a single point representing the center of each occupied voxel is preserved in the

401 point cloud while the extraneous points are filtered out.

402 Next, ground plane segmentation is performed using Random Sample Consensus

403 (RANSAC) [45]. Points from the ground plane are filtered out so that object points can be 
separated nicely in the clustering step. The RANSAC algorithm iteratively computes an estimate

405 of the ground plane parameters by randomly sampling points from the point cloud. The best

406 estimate in terms of number of inliers is determined and all points located within $0.4 \mathrm{~m}$ of the

407 calculated plane are filtered out.

The ensuing clustering step involves grouping points that are contained within the same

neighborhood together and labelling each group as an individual cluster. The threshold parameter used in this study is $0.4 \mathrm{~m}$ but it can also be manually adjusted so that the points belonging to the

411 same object would not be split into separate clusters and the points belonging to different objects

412 would not be grouped into the same cluster. Even with carefully tuned clustering parameters, this

413 step may occasionally face problems with over-segmentation or under-segmentation, which

414 affects the tightness of fit and orientation accuracy of the bounding boxes. However, the final

415 bounding box span in 3D space to determine collisions remains largely the same.

Finally, an oriented bounding box is computed for each point cluster by considering the

417 physical spread of points in the z-axis (vertical axis) and the $\mathrm{x}-\mathrm{y}$-plane. Principal Component

418 Analysis (PCA) [46] is used to determine the two principal directions in which points in the

419 cluster vary the most in the $x-y$-plane. The principal directions are used to determine the

420 orientation of the objects bounding box in the $x-y-p l a n e$. The final bounding box is then

421 computed by determining the maximum and minimum length along the vertical axes and the

422 horizontal principal axes. The total computation time for the whole process largely depends on

423 the down-sampling ratio but it only takes a few minutes to down sample a site point cloud of

424 around 30 million points. Regardless of the original point cloud resolution, a final point cloud

425 with a size of around 10,000 points is considered sufficient and efficient for obstruction

426 identification since it is only necessary to recover a rough outline of each detected object. 
Once the bounding boxes for obstructions are automatically created, they are prepared for

428 visualization with the adjustment of the surface transparency level and being labeled as

429 obstructions in a game engine. The framework for lift scene reconstruction is illustrated in Figure

4. As the obstructions in the as-is lift site are represented by bounding boxes, the site condition

431 can be easily updated based on actual changes by manually moving or adding bounding boxes

432 through the user interface. The recognized obstructions represent the possible collision risks on

433 the lift site that will be addressed in pro-active hazard analysis and visualization in real-time.
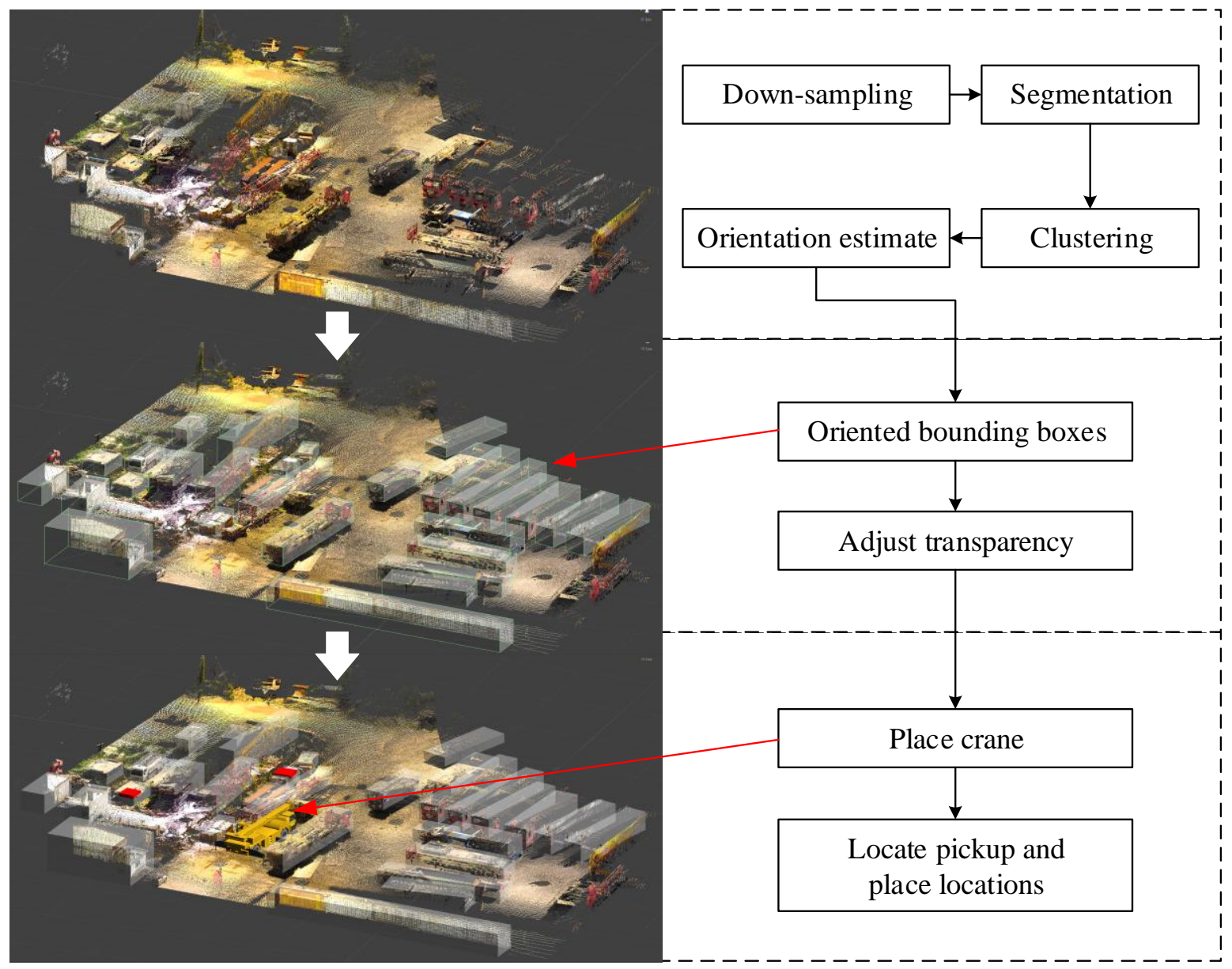

435 Figure 4: Lift site condition modeling process based on point cloud data 


\subsection{Pro-active Hazard Analysis and Real-time Visualization}

441 Once crane motions are captured and a lift site is reconstructed with the recognized obstructions,

442 the next task in this framework is to analyze potential hazards in real-time based on a pre-defined 443 proximity threshold. The pro-active hazard analysis is enabled by the four steps as follows. First, 444 the algorithm considers four crane parts as the objects subject to collision hazards: crane cabin, 445 boom, hoist line, and load. Second, the obstructions present on the site are categorized into three 446 levels of severity based on the potential consequences if the collision occurs. For example, power 447 lines in the crane workspace fall into the highest level of severity while trees fall into the lowest 448 level of severity. In the third step, based on safety regulations from the Occupational Safety and 449 Health Administration (OSHA) [47] and industry best practices [7], different safe proximity 450 thresholds are assigned to each severity levels as shown in Table 1. Lastly, the game engine 451 keeps tracks of the proximity between each crane part and each obstruction. The proximity is 452 defined as the distance between the closest points on the surfaces of each object. To minimize 453 the delay in real-time visualization, the hazard analysis needs to be conducted in an efficient manner. As such, the program only monitors the crane parts and the obstructions within a distance less than $5 \mathrm{~m}$; this input can be changed based on size of crane, types of lifting work, and conditions of job site. When the proximity between the crane part and the obstruction is

457 beyond the respective threshold, the program will highlight the objects of interest and send visual and auditory warnings through the graphical user interface to the operator.

Table 1: Proximity thresholds in the hazard analysis based on level of severity

\begin{tabular}{|l|l|l|}
\hline Level of severity & Proximity threshold & Examples \\
\hline High & $4 \mathrm{~m}$ & Energized power lines \\
\hline Moderate & $2 \mathrm{~m}$ & Building structures, equipment \\
\hline Low & $1 \mathrm{~m}$ & Trees \\
\hline
\end{tabular}




\section{PROTOTYPE DEVELOPMENT}

462 To validate the proposed framework, a prototype system was developed and deployed on a 70-

463 ton telescopic boom mobile crane. This section introduces how each part of the framework was

464 implemented and the performance of the prototype system was evaluated in field tests.

\subsection{Sensor Deployment}

467 The prototype system adopted rotary magnetic encoders to measure three critical motions: boom

468 slew, hoist line extension and boom extension (see Figure 5). The boom slew angle was

469 measured by attaching a small measuring gear on the rotary encoder to the main slewing gear

470 underneath the crane cabin. Similar to the slewing angle, the length of the hoist line was

471 calculated based on rotation of the crane hoist winch measured by another rotary encoder. The

472 boom extension was obtained using another rotary encoder by measuring the length of a wire

473 with one end fixed at the middle of the boom and the other end attached to the tip of the boom.

474 Unlike other motion measurements, the boom lift angle was directly measured by an inclination

475 sensor attached to the crane boom. All the rotary encoders and inclination sensor were powered

476 by the crane battery and connected to a microcontroller. 


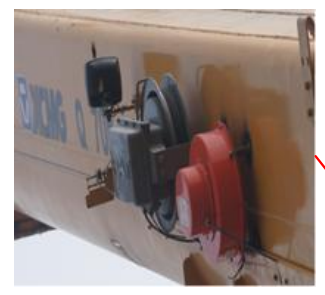

Boom length/angle (rotary encoder, inclination sensor)

Hoist line length (rotary encoder)
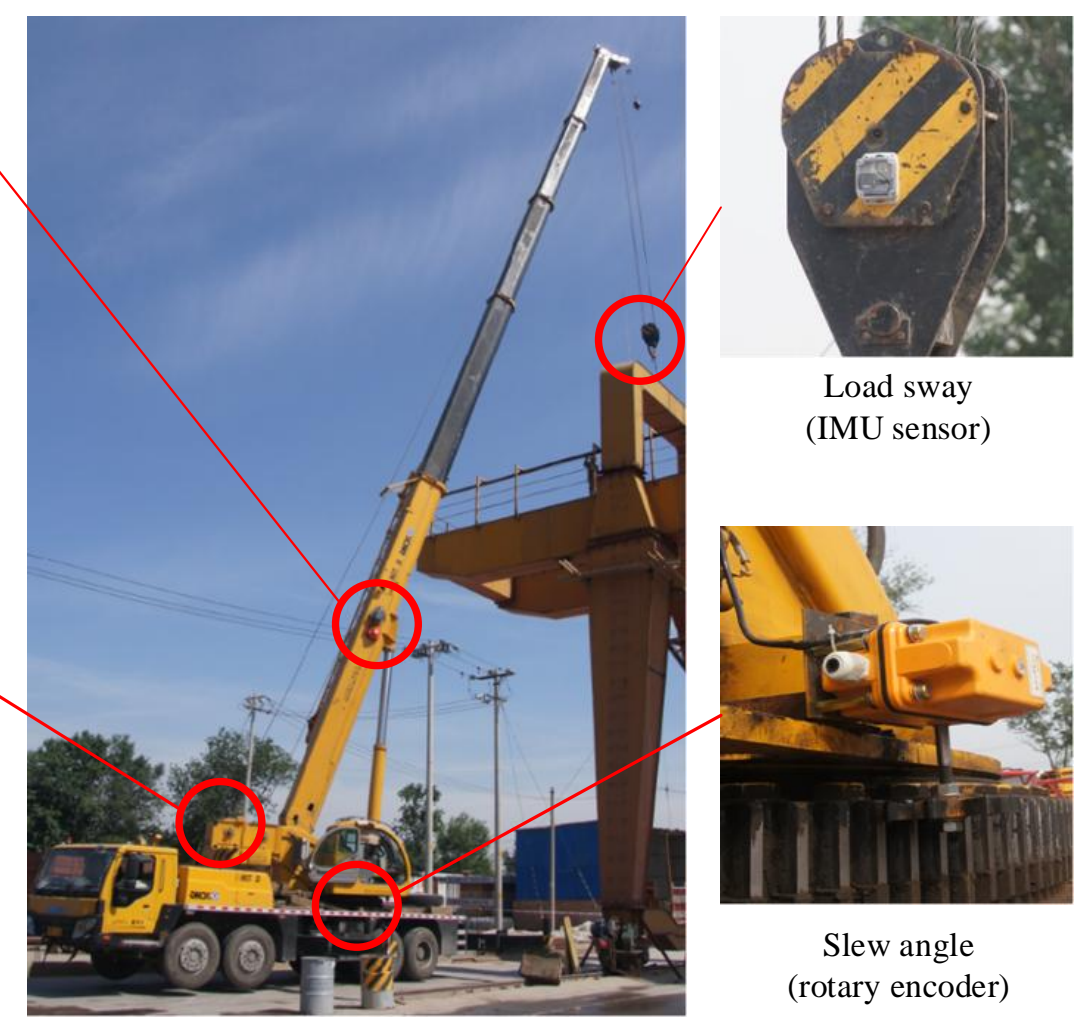

Load sway (IMU sensor)

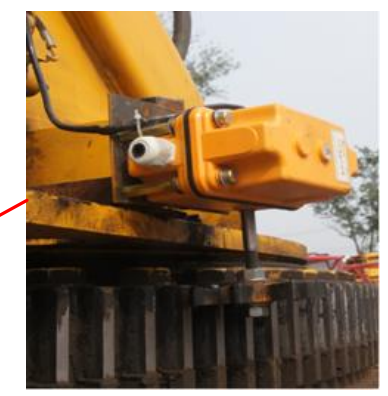

Slew angle (rotary encoder)

Figure 5: Sensor configuration for critical motion capturing

484 load orientation to the tablet for data processing. 


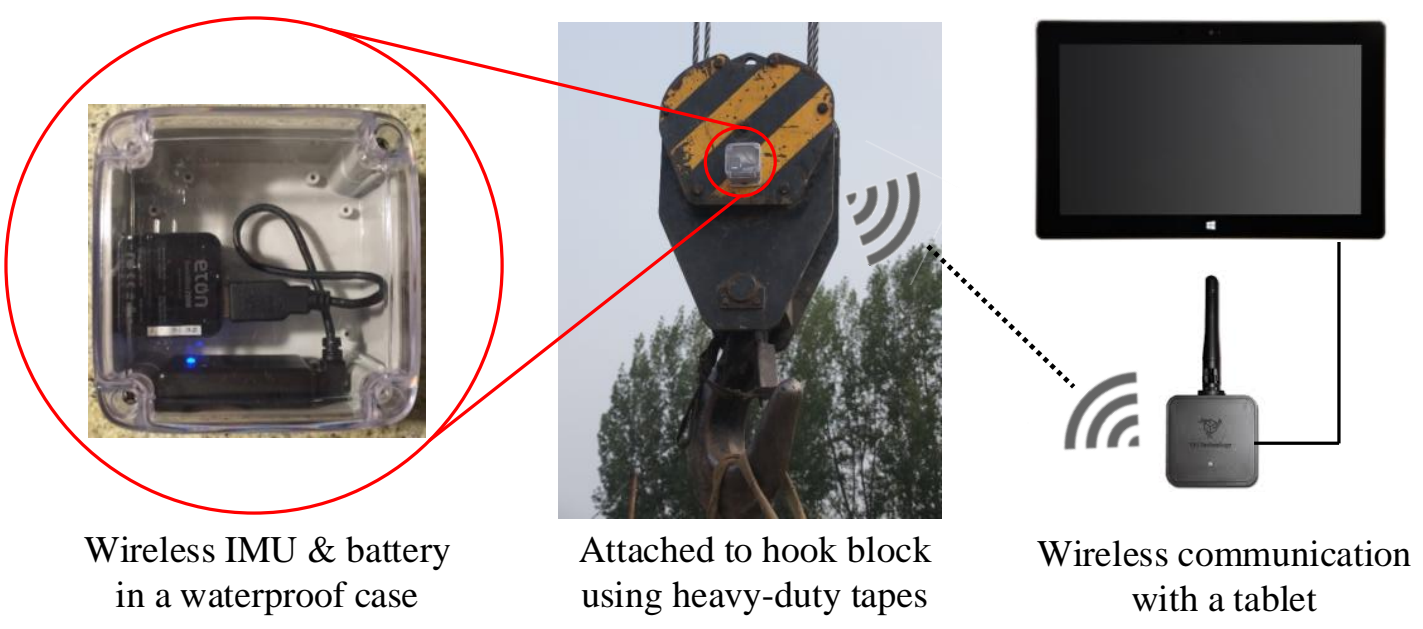

Figure 6: IMU sensor configuration for load position measurement

\subsection{Error Analysis of Crane Motion Reconstruction}

489 When analyzing the error of the final load positioning, error propagation as a result of the

changes in boom lift angle, slew angle, and boom extension must be considered. The goal of this

491 section is to understand how the error in the results of the pose reconstruction is affected by

Given that the load position is dependent on boom lift angle, boom length and boom slew angle,

494 a load position in X-Y plane can be calculated as shown in Eq. 2 and 3.

in which,

$$
\begin{aligned}
& x=L_{b} \cdot \cos \theta_{l} \cdot \sin \theta_{s} \\
& y=L_{b} \cdot \cos \theta_{l} \cdot \cos \theta_{s}
\end{aligned}
$$

$\theta_{l}:$ boom lift angle

$\theta_{s}$ : boom slew angle

$L_{b}:$ boom length

Eq. 6 shows a general equation for error propagation. Given that $R=R(X, Y, \ldots)(\mathrm{X}$ and $\mathrm{Y}$ are different measurements) and $\delta X$ and $\delta Y$ (the uncertainty of measurement $\mathrm{X}$ and $\mathrm{Y}$ ), the

504 standard deviation of R can be calculated by a generic expression as shown in Eq.6. Since the 
measurement.

$\delta R=\sqrt{\left(\frac{\partial R}{\partial X} \cdot \delta X\right)^{2}+\left(\frac{\partial R}{\partial Y} \cdot \delta Y\right)^{2}+\cdots}$

511 respectively.

$512 \delta x=$

$$
\sqrt{\sin ^{2} \theta_{l} \cdot \sin ^{2} \theta_{s} \cdot L_{b}{ }^{2} \cdot \delta \cos \theta_{l}{ }^{2}+\cos ^{2} \theta_{l} \cdot \cos ^{2} \theta_{s} \cdot L_{b}{ }^{2} \cdot \delta \sin \theta_{s}{ }^{2}+\cos ^{2} \theta_{l} \cdot \sin ^{2} \theta_{s} \cdot \delta L_{b}{ }^{2}+\delta x_{l}{ }^{2}}
$$

$\delta y=$

$\sqrt{\sin ^{2} \theta_{l} \cdot \cos ^{2} \theta_{s} \cdot L_{b}{ }^{2} \cdot \delta \cos \theta_{l}{ }^{2}+\cos ^{2} \theta_{l} \cdot \sin ^{2} \theta_{s} \cdot L_{b}{ }^{2} \cdot \delta \sin \theta_{s}{ }^{2}+\cos ^{2} \theta_{l} \cdot \cos ^{2} \theta_{s} \cdot \delta L_{b}{ }^{2}+\delta x_{l}{ }^{2}}(6)$

518 Thus, the error of load positioning in the X-Y plane can be obtained by Eq. 7.

$\delta P(x, y)=\sqrt{\delta x^{2}+\delta y^{2}}=$

$$
\sqrt{\sin ^{2} \theta_{l} \cdot L_{b}{ }^{2} \cdot \delta \cos \theta_{l}{ }^{2}+\cos ^{2} \theta_{l} \cdot{L_{b}}^{2} \cdot \delta \sin \theta_{s}{ }^{2}+\cos ^{2} \theta_{l} \cdot \delta{L_{b}}^{2}+\delta x_{l}{ }^{2}+\delta y_{l}{ }^{2}}
$$

522 According to the sensor datasheet, the measurement error of boom lift and slew angle

523 is $\pm 0.5 \mathrm{deg}$, and the measurement error of boom length is $\pm 0.1 \mathrm{~m}$. Therefore, when boom lift

524 angle $\theta \in\left(0,75^{\circ}\right)$ and boom extension $L_{b} \in(11,45 m)$ (maximum range limits according to the

525 tested crane specification), the load positioning error in X-Y plane was calculated based on Eq. 7.

526 Figure 7 shows the load positioning errors in $\mathrm{X}-\mathrm{Y}$ plane as a result of the propagation of boom 
527 angle and length measurement errors in different boom slew angles (i.e., 0, 45, 90 degree). It

528 should be noted that the maximum error of load positioning occurs when the boom fully extends

529 (45m), boom lifts up to the maximum angle (75 degree), and boom slews to 90 or 270 degree

530 from the front. This result indicates that crane pose reconstruction is likely to be less accurate in

531 such configuration, and conservative adjustments such as increasing the proximity thresholds in

532 the hazard analysis should be undertaken.
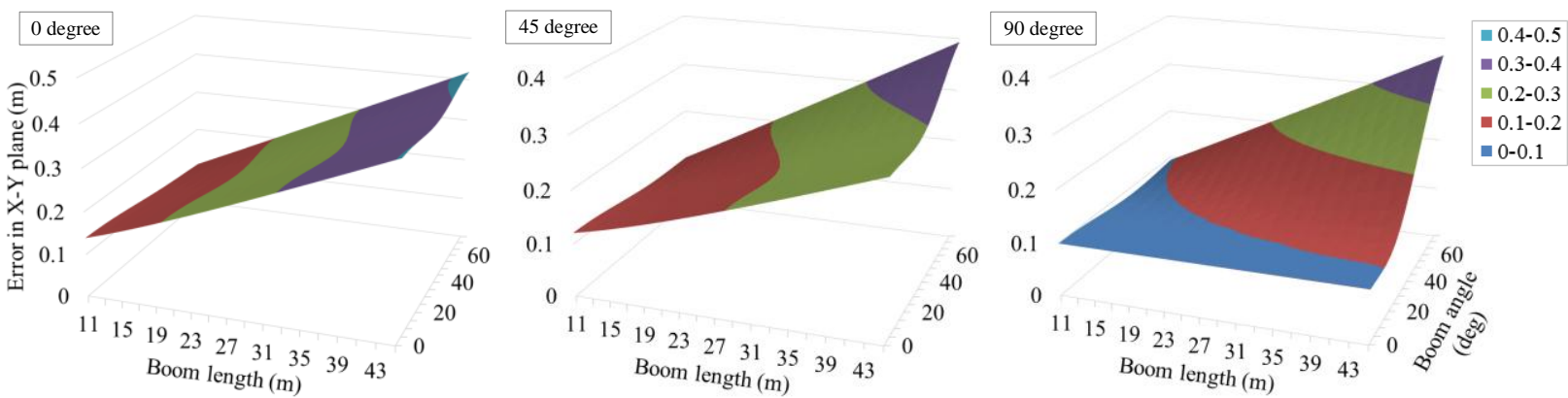

534 Figure 7: Load positioning errors in X-Y plane due to propagation of boom angle and length measurement errors, when boom slew angle is 0, 45 and 90 degree, respectively. processing unit so that the game engine program on the tablet receives the packet that includes

540 the measurements from all encoders at a frequency of $50 \mathrm{~Hz}$. In addition, the processing unit and

541 the program will detect and reject corrupted or incomplete packet. Given that all the encoders are

542 connected to the processing unit by cables, delay in data collection and transmission is negligible.

543 All these measures ensure the error related to data collection and synchronization is minimized.

\subsection{Operator User Interface}


In the prototype system, the tasks of hazard analysis and visualization were accomplished using

548 an on-board tablet computer. The tablet is equipped with an Intel Core i7 $1.7 \mathrm{GHz}$ processor, Intel

5495000 integrated graphic card, 8 GB RAM and 512 GB storage. The tablet offered a bright 11

550 inch display so that the user interface is visible to the operator even under normal sunlight

551 conditions (see Figure 8). The tablet was mounted on a fully adjustable monitor holder, which

552 makes it very flexible to be configured to what the operator considers fit. A game engine Unity

553 3D [48] was used as the program for real-time data processing and 3D visualization. To

554 minimize the distraction to the main lifting tasks, A commercial voice recognition program was

555 integrated into Unity3D to enable voice control over the user interface.

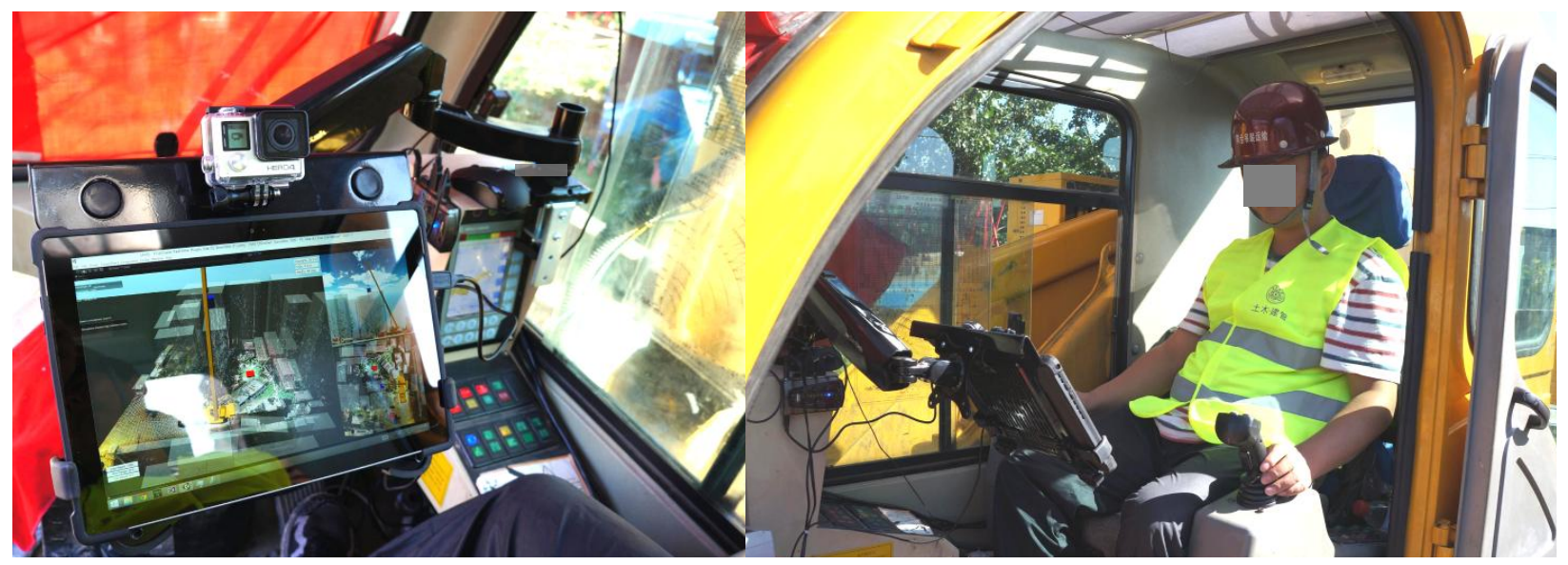

557 Figure 8: Graphical user interface deployed in a mobile crane cabin

\section{FIELD TESTS AND RESULTS}

\subsection{Overview}

561 The performance of the prototype system was evaluated in a field test. The prototype system was

562 installed on a 70-ton telescopic boom all-terrain crane manufactured by XCMG Construction

563 Machinery. The objective of the test was to evaluate the performance of the prototype system in

564 the criteria of reconstruction accuracy, latency and ease of use. The field test took place in a 
crane parts storage yard congested with obstructions such as truck trailers, lattice boom segments and trees as shown in Figure 9(a). Crane parts such as lattice boom segments and truck trailers were introduced in the scene to simulate the condition in a congested construction site. Figure 9(b) shows the top view of the reconstructed test site with obstructions indicated by the semitransparent boxes. The two dots indicate the load pick up and placement locations.
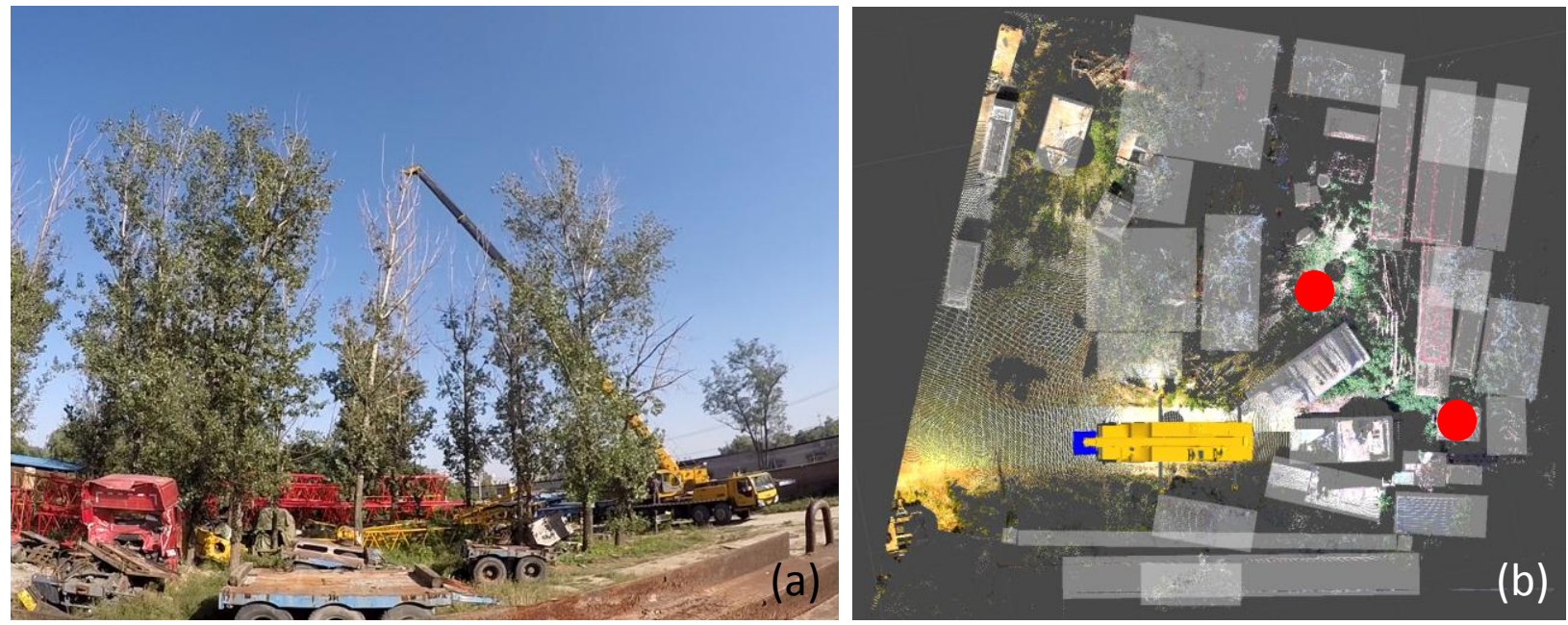

572 Figure 9: Actual test site and top view of the reconstructed test site (two dots indicate pick up 573 and drop off locations)

575 point cloud consists of 27 million points, from which 29 obstructions were automatically

576 modeled and three obstructions were manually modeled. The entire process including laser

577 scanning, obstruction recognition, and virtual lift site reconstruction took in total 35 minutes. 
582 The tablet served as a computation device for hazard analysis while displaying the reconstruction

583 lift site to the operator. Although visualization delay was expected because of data transmission

584 and computation, the test operators observed no noticeable delay during the test. The

585 visualization results were validated by comparing the reconstructed crane boom and load

586 motions to site camera recordings from two view angles: site overview and top view from the

587 crane boom head. The site overview comparison demonstrates the accuracy in reconstructing

588 crane boom motions (see Figure 10) and the top view from boom head comparison demonstrates

589 the accuracy in reconstructing crane load sway motions (see Figure 11). In these figures, the

590 solid cube indicates the lifted load and the circle indicates the target drop off location.

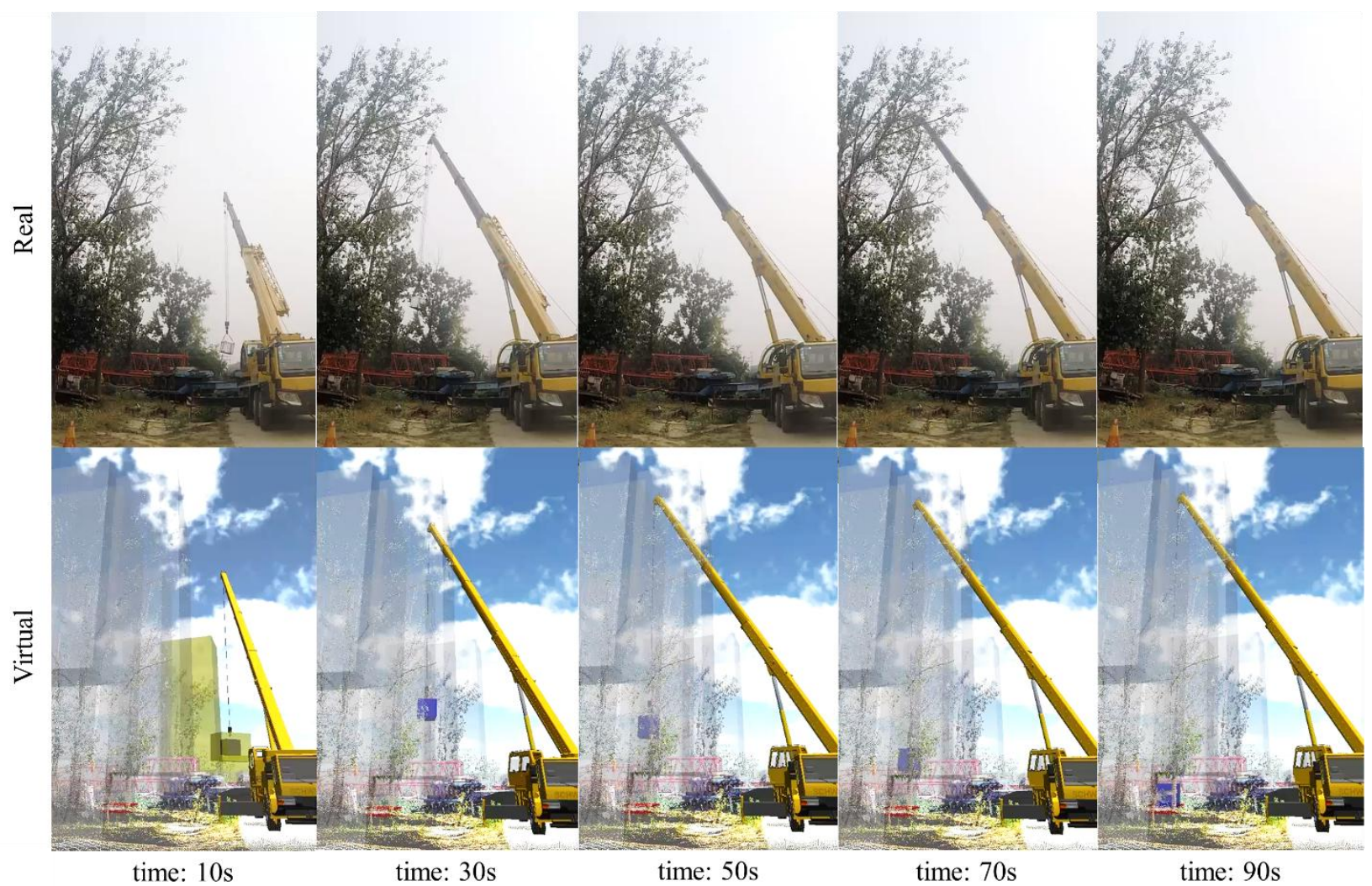

592 Figure 10: Comparison between actual lifting (upper) and reconstructed lifting process (lower) in 593 site overview 


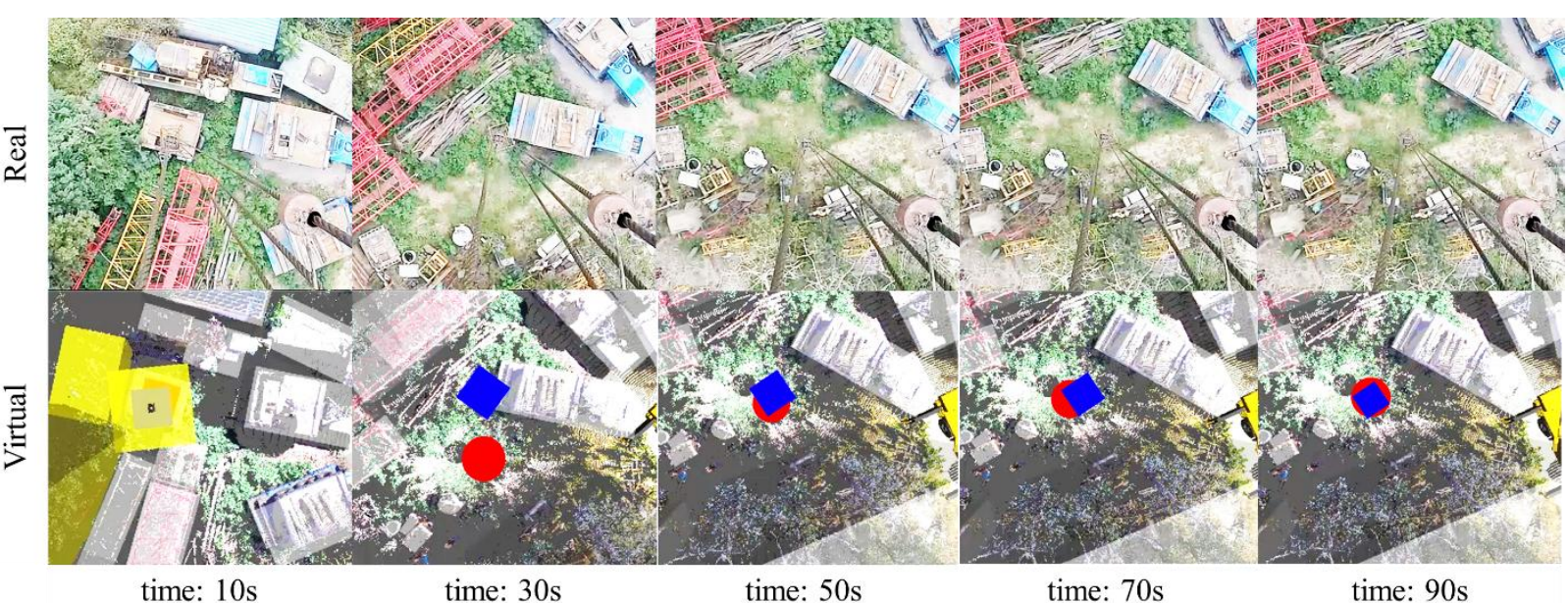

Figure 11: Comparison between actual load sway motion (upper) and reconstructed load sway motion (lower) from the boom's top view.

\subsection{Demonstration of Pro-active Safety Assistance}

The comparison between the actual crane motion and the reconstruction motion reveals the this hazard was detected, visual and audio warnings were immediately provided to the operator, and the objects involved the collision risk (i.e., lifted load and nearby trees) were automatically highlighted in the system user interface. This allowed the operator to pro-actively review and

608 assess the lift condition in the side and top views, or alternatively move the virtual camera by

609 voice commands to zoom in to the area of interest for a more effective judgement. 


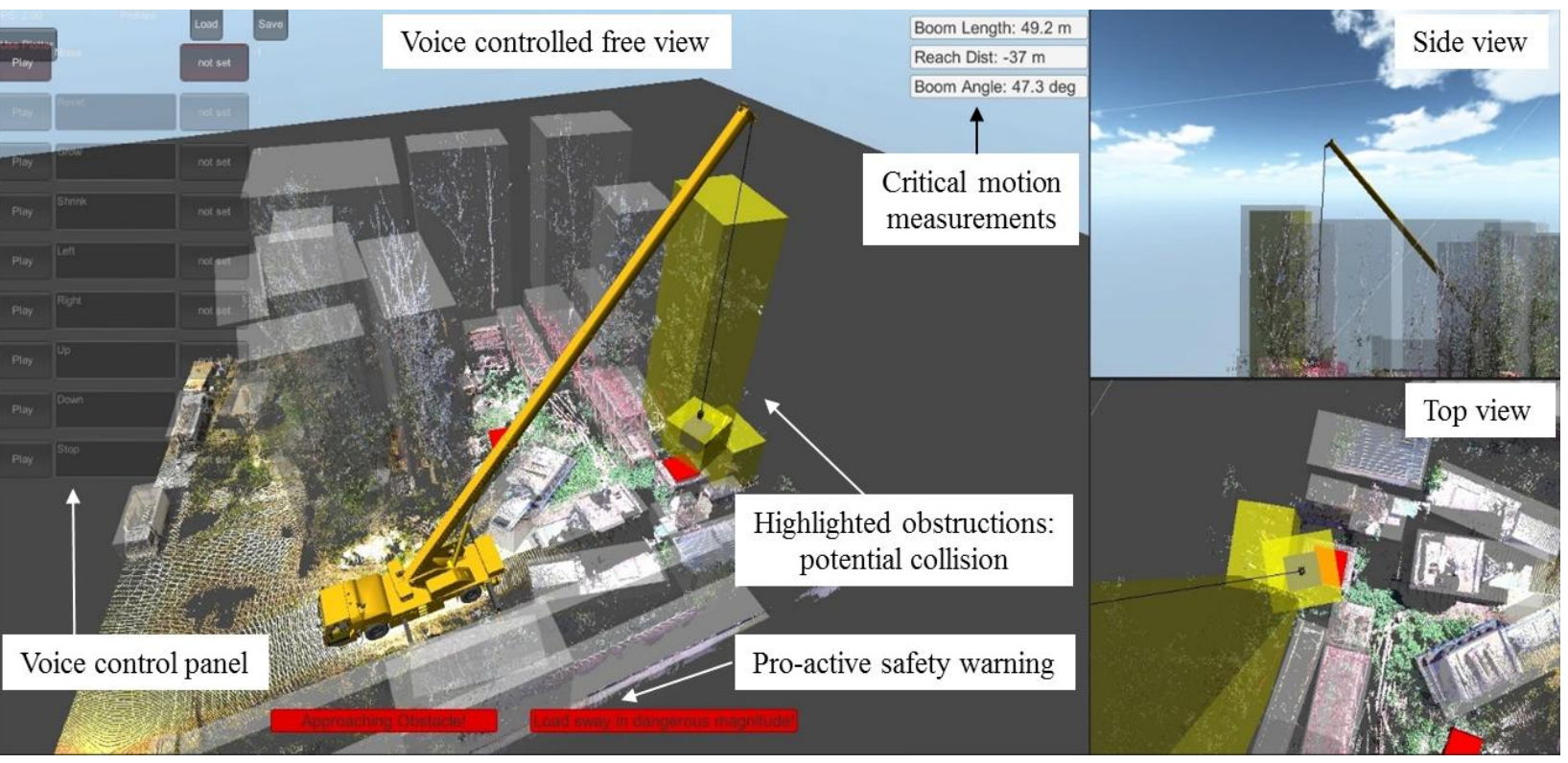

612 Figure 12: User interface of the pro-active safety assistance system

\section{5.4. Reconstruction Error Analysis}

615 Since it is very difficult to measure the error during the lift, the overall reconstruction error of the

616 system was represented by the error of load positioning at the end of each lift. This assumption is

617 reasonable as the final load position is greatly affected by the reconstruction accuracy of both the

618 boom and load motion. Furthermore, it also reflects the overall error that may accumulate during

619 the lift. As the load was always placed at the exact same drop off location, the load positioning

620 error is the horizontal distance between the actual and planned drop off location. Figure 13 plots

621 the load positioning errors in 92 consecutive lifts, and the maximum, minimum, median, first and

622 third quartile of the errors are shown in the box plot to the right. The average error for

623 reconstruction is $0.43 \mathrm{~m}$ and the maximum error for reconstruction is $0.93 \mathrm{~m}$. Compared to

624 theoretical error calculated based on the propagation of nominal sensor errors, the average value

625 of the actual errors is slightly higher $(0.43 \mathrm{~m}$ vs. $0.38 \mathrm{~m})$ and the variances are bigger as well

$626(0.49 \mathrm{~m}$ vs. $0.21 \mathrm{~m})$. Differences between the actual and theoretical errors are mainly due to 
627 variances in sensor setup or system calibration as well as possible measurement errors. The

628 logarithmic trend line indicates a slight increasing tendency of the errors over the 92 lifts. It

629 should be noted that no re-calibration was conducted during the test lifts. This indicates the

630 system error did accumulate over time in extensive usage conditions, and thus re-calibration is

631 necessary in actual field implementation in the long-term.

632

633

634

635

636

637

638

639

640 641 effective in reconstructing the lift scene and providing them hazard warnings. Based on this

642 information, they were able to make timely decisions to mitigate the risk. Overall, the operators

643 believed that using this system would make them feel more confident to execute the lift task

Figure 13: Errors of virtual lift reconstruction during 92 lift tasks in scenario 1 and 2

\subsection{Operator Feedback}

In addition to testing the technical performance, it is important to know how much the operators are receptive to the new system in terms of effectiveness of the assistance and user friendliness. In this test, five crane operator subjects with an average experience of 18 years in crane operation were invited to test the prototype system and their feedback toward the current prototype system is listed in Table 2. Most of the operators expressed that the system was

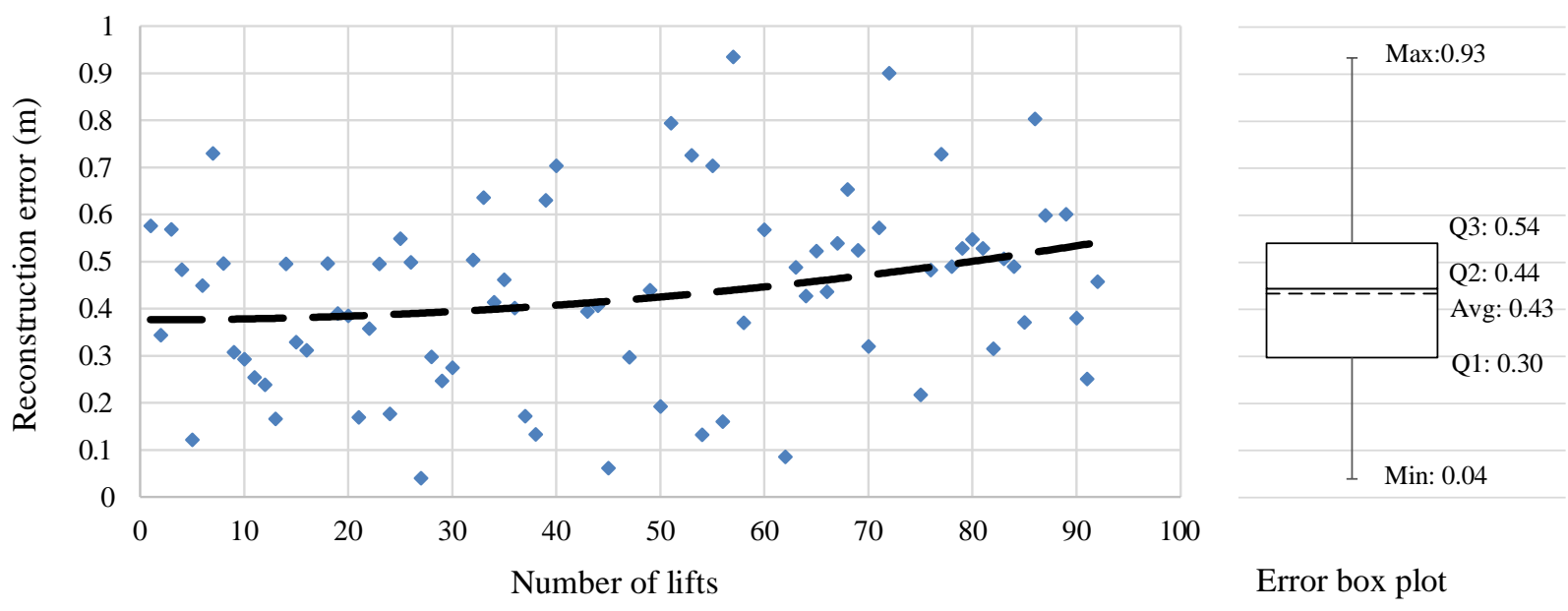


644 more efficiently and safely. In addition, three operators pointed out that this system is

645 particularly useful in blind lift scenarios where the operator can review the constraints in the lift 646 and make the right decisions instead of totally relying on the signalman.

648 Table 2: Feedback from subject tests on the performance of the prototype system

\begin{tabular}{|c|c|c|}
\hline Feedback & Positive & Negative \\
\hline $\begin{array}{l}\text { Operator A } \\
\text { (15-year exp.) }\end{array}$ & $\begin{array}{l}\text { - I am already relying on this system } \\
\text { - Reconstruction is fast and accurate }\end{array}$ & $\begin{array}{l}\text { - System setup take quite a while } \\
\text { - Better have a shade for the screen }\end{array}$ \\
\hline $\begin{array}{l}\text { Operator B } \\
\text { (13-year exp.) }\end{array}$ & $\begin{array}{l}\text { - Very useful for night lifting tasks } \\
\text { - I can totally trust this system }\end{array}$ & $\begin{array}{l}\text { - Top view is more useful, should be enlarged } \\
\text { - Should display the motion data from LMI }\end{array}$ \\
\hline $\begin{array}{l}\text { Operator C } \\
\text { (20-year exp.) }\end{array}$ & $\begin{array}{l}\text { - Very useful for blind and night lifts } \\
\text { - It makes me feel safer }\end{array}$ & - Orientation should be shown more clearly \\
\hline $\begin{array}{l}\text { Operator D } \\
\text { (21-year exp.) }\end{array}$ & $\begin{array}{l}\text { - It can already replace the LMI } \\
\text { system }\end{array}$ & $\begin{array}{l}\text { - It should show the distance between load } \\
\text { and obstructions and drop-off location }\end{array}$ \\
\hline $\begin{array}{l}\text { Operator E } \\
\text { (19-year exp.) }\end{array}$ & - Very useful for blind and night lifts & \\
\hline
\end{tabular}

\section{DISCUSSION}

651 Accurate motion capturing and reconstruction are essential for enabling real-time hazard analysis

652 and visualization. The error of load positioning mainly comes from three sources: sensor

653 measurement error, error propagation in motion reconstruction, and accumulated system error

654 due to sensor setup. Sensor measurement error can be reduced by using sensors that are more

655 accurate and durable. Error propagation in motion reconstruction varies in different crane

656 configurations (e.g., boom length and angle) and thus can be hard to reduce. The accumulated

657 system error can be reduced by improving the way the sensors are deployed. For example, the

658 length of the hoist line is measured by attaching a small wheel to the edge of the hoist line winch.

659 Although the contact between the wheel and the winch is perfectly firm in the beginning, it 
660 might become loose in the long term and thus increase the error in the length measurement of the

661 hoist line. This problem can be solved by deploying a rotary encoder directly on the axle on the

662 winch with an enclosure. Such improvement in system deployment will be addressed in the

663 future study.

664 Another critical issue that can significantly compromise the effectiveness of real-time

665 systems is the delay or latency in data processing and visualization. Crane lifting operations are

666 so dynamic that an accident can develop in a couple of seconds. The tested prototype system

667 showed no noticeable delay or latency based on the operator feedback and the comparison

668 between the actual and reconstructed crane motion (see Figure 9 and 10). This is partially

669 because the communication between sensors (except the IMU sensor) and the computer were via

670 wired connection. In addition, delay in data processing or lagging in visualization can be

671 expected if a less powerful tablet is used for data processing and visualization.

672 Ideally, the site condition is preferred to be updated in real-time to capture any tiny

673 changes at any given moment. In reality, technology limitations make real-time 3-dimension

674 geometry updating very challenging and expensive even if possible. At the same time, it is not

675 always necessary to update the site condition in real-time. First, workers as the most dynamic

676 assets on construction site, does not need to be tracked on the lift site considering that workers

677 except the lift crew are not allowed to be in the lift zone. Secondly, although other mobile asset

678 such as equipment and vehicles might be present in the lift workspace for material delivery, their

679 location and movement are fairly predictable and relatively static during the lifting operation. As

680 such, frequent updates for the site condition, as needed, is regarded sufficient to address the

681 changes on the lift site. That said, the site condition should be automatically updated as a new

682 change is introduced. Although this is beyond the scope of this paper, promising strategies for 
683 real- or near real-time lift site condition updating are discussed as below. As stated before, laser

684 scanning is the most efficient method for acquiring a large amount of 3D geometric data in a

685 short period of time. Many state-of-the-art laser scanners can be remotely operated and

686 wirelessly transfer the scan results to remote computers for processing. Therefore, such terrestrial

687 laser scanners can continuously scan the lift site and update the condition changes with the

688 obstruction recognition algorithm. As a future study, the authors are planning to use a mobile

689 robot equipped with a laser scanner to add a mobility of scanner for a better scan view for the

690 changes of the lift site.

691

\section{7. CONCLUSIONS}

693 Despite many safety considerations embedded in lift pre-planning, the ability to provide real-

694 time safety assistance to crane operators during the lifting is missing in the state-of-the-art. This

695 paper introduces a framework of a safety assistance system for crane lift operations. The

696 framework consists of three components: real-time crane motion monitoring, pro-active hazard

697 analysis and 3D visualization. Critical motions of crane manipulation and suspension modules

698 are captured by a hybrid sensor structure, based on which entire crane motions are reconstructed

699 in a visualization platform in real-time with as-is lift site conditions. The risk of colliding the

700 boom and load to obstructions in the lift site is analyzed pro-actively and warnings are provided

701 to operators when a potential hazard is detected. The framework was validated by a prototype

702 deployed on a real crane and a field test was conducted to evaluate the system performance with

703 respect to reconstruction accuracy, timing, hazard analysis and visualization effectiveness. Test

704 results indicate that the prototype system was able to reconstruct crane motions with an average

705 error of $0.43 \mathrm{~m}$ for load positioning in $\mathrm{X}-\mathrm{Y}$ plane. It also proved that the system is effective in 
providing pro-active hazard warnings that allows the operator to make timely decisions to mitigate the risk. The feedback from five experienced test subjects show a great potential of the pro-active safety assistance system in enhance the existing lift safety practices, especially in blind and night lifts. This paper broadens the focus of crane lift safety from pre-planning phase to the operation phase. The framework introduced in this paper successfully addresses various challenges in enabling real-time pro-active safety assistance in crane lifting operations. The error analysis approach is useful for not only crane monitoring systems, but also other construction heavy equipment.

\section{ACKNOWLEDGEMENT}

This material is based upon work supported by the National Science Foundation (Award \#:

CMMI-1358176). Any opinions, findings, and conclusions or recommendations expressed on this material are those of the authors and do not necessarily reflect the views of the NSF.

\section{REFERENCES}

[1] S. Kang, E. Miranda, Planning and visualization for automated robotic crane erection processes in construction, Automation in Construction 15 (2006) 398-414. doi:10.1016/j.autcon.2005.06.008.

[2] J.E. Beavers, F. Asce, J.R. Moore, R. Rinehart, W.R. Schriver, Crane-Related Fatalities in the Construction Industry, Journal of Construction Engineering and Management (2006) 901-910. doi:10.1061/(ASCE)0733-9364(2006)132:9(901).

[3] CPWR, Crane-Related Deaths in Construction and Recommendations for Their Prevention, 2009. http://www.cpwr.com/sites/default/files/CPWR Crane Rept Recmmdtns Nov-2009-BLS UPDATED.pdf.

[4] R.L. Neitzel, N.S. Seixas, K.K. Ren, A review of crane safety in the construction industry, Applied Occupational and Environmental Hygiene 16 (2001) 1106-17. doi:10.1080/10473220127411.

[5] A. Suruda, M. Egger, D. Liu, Crane-Related Deaths in the U.S. Construction Industry, 1984-94, Journal of Construction Engineering and Management 132(9) (1997) 901-910. 
[6] K. Tantisevi, B. Akinci, Automated generation of workspace requirements of mobile crane operations to support conflict detection, Automation in Construction 16 (2007) 262276. doi:10.1016/j.autcon.2006.05.007.

[7] L.K. Shapiro, J.P. Shapiro, Cranes and Derricks 4th Edition, McGraw-Hill, New York, 2011. ISBN: 0071625577

[8] R.A. King, Analysis of Crane and Lifting Accidents in North America from 2004 to 2010, (2012). https://dspace.mit.edu/handle/1721.1/73792 (accessed August 19, 2016).

[9] J.G. Everett, A.H. Slocum, Cranium: Device for Improving Crane Productivity and Safety, Journal of Computing in Civil Engineering 119 (1993) 23-39. doi:10.1061/(ASCE)07339364(1993)119:1(23).

[10] Y.K. Cho, C.T. Haas, Rapid geometric modeling for unstructured construction workspaces, Computer-Aided Civil and Infrastructure Engineering 18 (2003) 242-253. doi:10.1111/1467-8667.00314.

[11] Y.K. Cho, C.T. Haas, K. Liapi, S. V. Sreenivasan, A framework for rapid local area modeling for construction automation, Automation in Construction 11 (2002) 629-641. doi:10.1016/S0926-5805(02)00004-3.

[12] W.C. Hornaday, C.T. Haas, J.T. O'Connor, J. Wen, Computer-aided planning for heavy lifts, Journal of Construction Engineering and Management 119 (1994) 498-515. doi:10.1061/(ASCE)0733-9364(1993)119:3(498).

[13] P. Zhang, F.C. Harris, P.O. Olomolaiye, G.D. Holt, Location Optimization for a Group of Tower Cranes, Journal of Construction Engineering and Management 1 (1999) 115-122. doi:10.1061/(ASCE)0733-9364(1999)125:2(115).

[14] V. Kamat, J. Martinez, Visualizing Simulated Construction Operation in 3D, Journal of Computing in Civil Engineering (2001) 329-337. doi:10.1061/(ASCE)08873801(2001)15:4(329).

[15] M. Al-Hussein, M. Athar Niaz, H. Yu, H. Kim, Integrating 3D visualization and simulation for tower crane operations on construction sites, Automation in Construction 15 (2006) 554-562. doi:10.1016/j.autcon.2005.07.007.

[16] M. Marzouk, A. Abubakr, Decision support for tower crane selection with building information models and genetic algorithms, Automation in Construction (2015). doi:10.1016/j.autcon.2015.09.008.

[17] K. Lin, C.T. Haas, Multiple heavy lifts optimization, Journal of Construction Engineering and Management 122 (1996) 354-362. doi:10.1061/(ASCE)0733-9364(1996)122:4(354).

[18] M. Al-Hussein, S. Alkass, O. Moselhi, Optimization Algorithm for Selection and on Site Location of Mobile Cranes, Journal of Construction Engineering and Management 131 (2005) 579-590. doi:10.1061/(ASCE)0733-9364(2005)131:5(579).

[19] C.M. Tam, T.K.L. Tong, GA-ANN model for optimizing the locations of tower crane and supply points for high-rise public housing construction, Construction Management and Economics 21 (2003) 257-266. doi:10.1080/0144619032000049665.

[20] Z. Lei, H. Taghaddos, U. Hermann, M. Al-hussein, A methodology for mobile crane lift path checking in heavy industrial projects, Automation in Construction 31 (2013) 41-53. 
doi:10.1016/j.autcon.2012.11.042.

[21] Y.-C. Chang, W.-H. Hung, S.-C. Kang, A fast path planning method for single and dual crane erections, Automation in Construction 22 (2012) 468-480. doi:10.1016/j.autcon.2011.11.006.

[22] J.J. Kuffner, S.M. LaValle, RRT-connect: An efficient approach to single-query path planning, Proceedings 2000 ICRA. Millennium Conference. IEEE International Conference on Robotics and Automation. Symposia Proceedings 2 (2000) 995-1001. doi:10.1109/ROBOT.2000.844730.

[23] Y. Lin, X. Wang, D. Wu, X. Wang, S. Gao, Lift Path Planning for Telescopic Crane Based-on Improved hRRT, International Journal of Computer Theory and Engineering 5 (2013) 816-819. doi:10.7763/IJCTE.2013.V5.803.

[24] C. Zhang, A. Hammad, Improving lifting motion planning and re-planning of cranes with consideration for safety and efficiency, Advanced Engeering Informatics 26 (2012) 396410. doi:10.1016/j.aei.2012.01.003.

[25] H. AlBahnassi, A. Hammad, Near real-time motion planning and simulation of cranes in construction: Framework and system architecture, Journal of Computing in Civil Engineering 26 (2012) 54-63. doi:10.1061/(ASCE)CP.1943-5487.0000123.

[26] J. Reason, Human Error, Cambridge University Press, 1990. ISBN: 0521314194.

[27] Occupational Safety and Health Administration, Cranes and Derricks in Construction; Final Rules, 2010. https://www.osha.gov/FedReg_osha_pdf/FED20100809.pdf.

[28] U.-K. Lee, K.-I. Kang, G.-H. Kim, H.-H. Cho, Improving Tower Crane Productivity Using Wireless Technology, Computer-Aided Civil and Infrastructure Engineering 21 (2006) 594-604. doi:10.1111/j.1467-8667.2006.00459.x.

[29] A. Shapira, F. Asce, Y. Rosenfeld, I. Mizrahi, Vision System for Tower Cranes, Journal of Construction Engineering and Management (2008) 320-332. doi:10.1061/(ASCE)07339364(2008)134:5(320).

[30] G. Putnik, V. Shah, V. Spasojević-Brkić, C. Alves, H. Castro, A proposal for installation architecture for video cameras and screens in an integrated vision system for crane cabins, 9th International Quality Conference (2015) 633-640.

[31] C. Zhang, A. Hammad, M. Asce, S. Rodriguez, Crane Pose Estimation Using UWB RealTime Location System, Journal of Computing in Civil Engineering (2012) 625-637. doi:10.1061/(ASCE)CP.1943-5487.0000172.

[32] S. Hwang, Ultra-wide band technology experiments for real-time prevention of tower crane collisions, Automation in Construction 22 (2012) 545-553. doi:10.1016/j.autcon.2011.11.015.

[33] H. Li, G. Chan, M. Skitmore, Integrating real time positioning systems to improve blind lifting and loading crane operations, Construction Management and Economics 31 (2013) 1-10. doi:10.1080/01446193.2012.756144.

[34] N. Li, B. Becerik-Gerber, Performance-based evaluation of RFID-based indoor location sensing solutions for the built environment, Advanced Engeering Informatics 25 (2011) 535-546. doi:10.1016/j.aei.2011.02.004. 
[35] Y.K. Cho, J.H. Youn, D. Martinez, Error modeling for an untethered ultra-wideband system for construction indoor asset tracking, Automation in Construction 19 (2010) 4354. doi:10.1016/j.autcon.2009.08.001.

[36] G. Lee, H.-H. Kim, C.-J. Lee, S.-I. Ham, S.-H. Yun, H. Cho, et al., A laser-technologybased lifting-path tracking system for a robotic tower crane, Automation in Construction 18 (2009) 865-874. doi:10.1016/j.autcon.2009.03.011.

[37] G. Lee, J. Cho, S. Ham, T. Lee, G. Lee, S.-H. Yun, et al., A BIM- and sensor-based tower crane navigation system for blind lifts, Automation in Construction 26 (2012) 1-10. doi:10.1016/j.autcon.2012.05.002.

[38] W. Ren, Z. Wu, Real-Time Anticollision System for Mobile Cranes during Lift Operations, Journal of Computing in Civil Engineering (2012) 1-12. doi:10.1061/(ASCE)CP.1943-5487.0000438.

[39] Wikipedia, Rotary encoder, (2016). https://en.wikipedia.org/wiki/Rotary_encoder (accessed August 19, 2016).

[40] Baumer Group, Cranes and Heavy Vehicles, (2016). http://www.baumer.com/fileadmin/user_upload/international/Downloads/BRCT/Baumer_Heavy-Vehicles-Cranes_BR_EN_1108_11047951.pdf (accessed August 19, 2016).

[41] Y. Fang, Y.K. Cho, Crane Load Positioning and Sway Monitoring Using an Inertial Measurement Unit, in: Computing in Civil Engineering 2015, Austin, TX, 2015: pp. 700707.

[42] FARO Technology Ltd., FARO Laser Scanner Focus 3D X330, (2015). https://www.reproproducts.com/pdfs/brochures/faro/x330.pdf (accessed August 19, 2016).

[43] C. Wang, Y.K. Cho, Smart scanning and near real-time 3D surface modeling of dynamic construction equipment from a point cloud, Automation in Construction 49 (2015) 239249. doi:10.1016/j.autcon.2014.06.003.

[44] Y.K. Cho, M. Gai, Projection-Recognition-Projection Method for Automatic Object Recognition and Registration for Dynamic Heavy Equipment Operations, Journal of Computing in Civil Engineering 28 (2014) A4014002. doi:10.1061/(ASCE)CP.19435487.0000332.

[45] R. Schnabel, R. Wahl, R. Klein, Efficient RANSAC for point-cloud shape detection, Computer Graphics Forum 26 (2007) 214-226. doi:10.1111/j.1467-8659.2007.01016.x.

[46] N. Locantore, J.S. Marron, D.G. Simpson, N. Tripoli, J.T. Zhang, K.L. Cohen, Robust principal component analysis for functional data, Test. 8 (1999) 1-73. doi:10.1007/BF02595862.

[47] Occupational Safety \& Health Administration, Cranes and Derricks in Construction (1926.1408), (2016). https://www.osha.gov/pls/oshaweb/owadisp.show_document?p_table=STANDARDS\&p_ $\mathrm{id}=19$ (accessed August 19, 2016).

[48] Unity Technologies, Unity3D, (2016). https://www.unity3d.com (accessed August 19, 2016). 

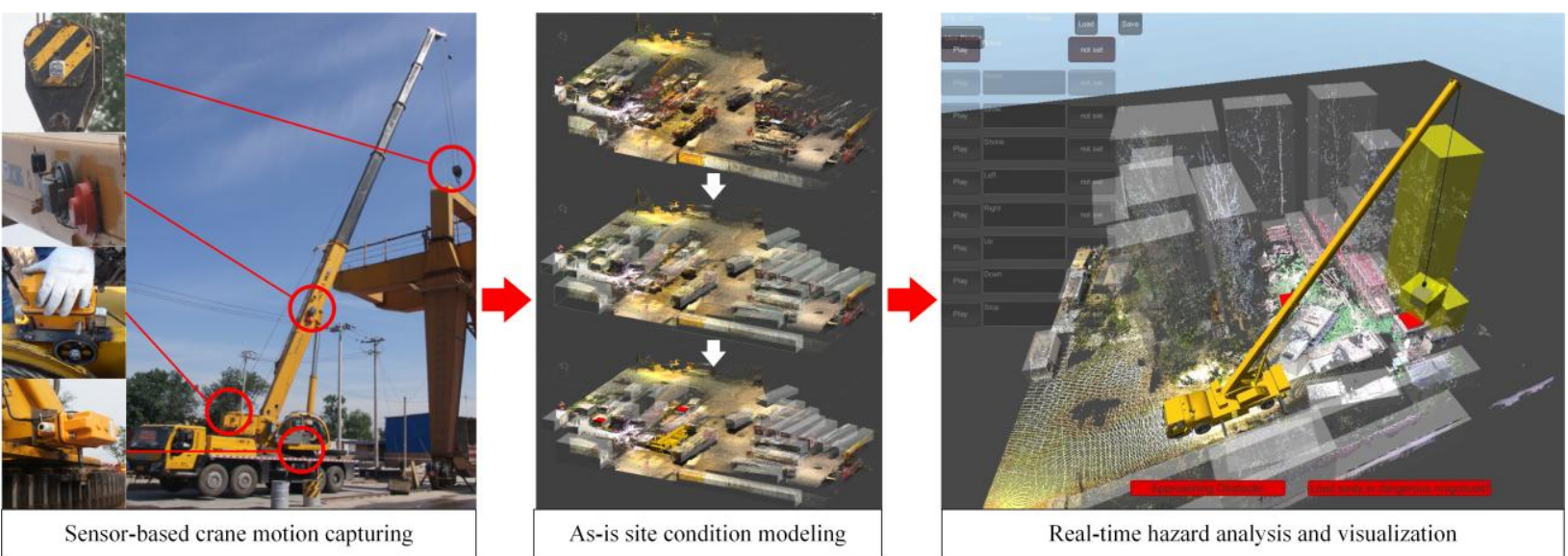

858

$$
\text { Sensor-based crane motion capturing }
$$

As-is site condition modeling

Real-time hazard analysis and visualization 